\title{
Acute pancreatitis and gastric dilatation in a patient with anorexia nervosa
}

\author{
S.A. Backett \\ Royal Edinburgh Hospital, Edinburgh EH10 5HF, UK.
}

\begin{abstract}
Summary: A case is described in which a young woman undergoing treatment for anorexia nervosa developed gastric dilatation and acute pancreatitis.

Two previous reports have described these as complications of anorexia nervosa and in view of this case attention is drawn to a possible association between these conditions. The importance of monitoring the anorectic patient's clinical state during the early phase of treatment is stressed.
\end{abstract}

\section{Introduction}

The consequences of even minor physical illness can be serious for patients debilitated after a period of prolonged malnourishment. Such a situation may be considered rare in the United Kingdom, but is observed in patients with anorexia nervosa.

The early stage of treatment, when feeding patterns are being re-established, is often accompanied by vague and non-specific abdominal symptoms. The precise origin of these symptoms is often difficult to determine, and in the majority of cases may be selflimiting. As a result of this a psychogenic explanation may be invoked. It is important to be aware that in some instances these symptoms herald the onset of a complication which if not diagnosed and treated promptly may have serious consequences. A case is described in which these events occurred.

\section{Case history}

The patient was a seventeen year old schoolgirl. She came from a closely knit middle class family and was the second oldest of three children, having a sister two years older than her and a brother three years younger.

A year before she was referred for treatment with anorexia nervosa her brother had been diagnosed as diabetic. She was reported to have studied his diet sheets and shortly after this she herself started to reduce her food intake by limiting the amount of carbohydrate she ate. Over the months that followed the range of foodstuffs eaten became increasingly

S.A. Backett, M.R.C.Psych., M.Phil. Accepted: 29 November 1983 limited and there was evidence of ritualistic behaviour associated with meals. She was noted to be unhappy and irritable and to have withdrawn from social contacts outside the family. Instead she became excessively dependent upon her mother and appeared reluctant to be separated from her. Throughout this time there was no evidence of bulimia, self-induced vomiting or abuse of purgatives. In nine months she lost $17 \mathrm{~kg}$ and became amenorrhoeic. She was finally taken by her parents to her general practitioner, who referred her for psychiatric assessment, from where she was admitted for in-patient treatment.

On admission to hospital she was found to be $161 \mathrm{~cm}$ tall and weighed $31 \mathrm{~kg}$. Physical examination revealed signs of gross emaciation. She was hypotensive and had a pulse rate of 40 beats $/ \mathrm{min}$. Her blood urea was elevated at $16.1 \mathrm{mmol} / \mathrm{l}$. All other haematological investigations including haemoglobin, electrolytes and blood sugar were within normal limits. An electrocardiogram confirmed the presence of sinus bradycardia.

Her initial diet consisted predominantly of liquids in the form of milk puddings and eggs. After 3 days solids were introduced and in the two weeks that followed a fairly normal eating pattern was re-established. Her weight increased by $2.5 \mathrm{~kg}$. No medication other than vitamin supplements was given. On the fifteenth day following her admission she complained of mild abdominal discomfort, which gradually became more severe as the day progressed and was associated with nausea and vomiting. During the night that followed her condition suddenly deteriorated and reassessment of her physical state at this time revealed a distressed young woman, with a pulse rate of 130 beats/min, and

(C) The Fellowship of Postgraduate Medicine, 1985 
blood pressure of $80 / 60 \mathrm{~mm} \mathrm{Hg}$. Her abdomen was rigid and bowel sounds absent. She was transferred to a surgical unit in the nearby general hospital and a further assessment of her clinical state was made.

A straight X-ray of her abdomen showed marked dilatation of both the stomach and duodenal cap and further dilatation of the small and large bowel. Blood urea and electrolytes, liver function tests, calcium and phosphate were all within normal limits. Her serum amylase was elevated at $1535 \mathrm{U} / 1$ (normal range 50$300 \mathrm{U} / \mathrm{l}$ ). A diagnosis of acute pancreatitis was made and she was managed conservatively with gastric suction and intravenous infusion. With the passage of the nasogastric tube there was relief of the gastric distension with a dramatic improvement in her clinical state. Oral fluids were reintroduced four days after her admission. Her progress continued in an uneventful manner and two weeks later she was transferred back to the psychiatric hospital where she remained for the next two months. She was discharged from in-patient care weighing $46 \mathrm{~kg}$ and was then followed up as an out-patient.

\section{Discussion}

Several authors have reported gastric dilatation and duodenal ileus as a complication of anorexia nervosa (Russell, 1966; Evans, 1968; Scobie, 1973; Brook, 1977). Serious consequences from the dilatation itself are rare except when it leads to spontaneous rupture of the stomach, a condition which is associated with appreciable mortality (Evans, 1968). Suggested mechanisms for the development of this dilatation are numerous. Two previous reports have linked acute

\section{References}

BROOK, G.K. (1977). Acute gastric dilatation in anorexia nervosa. British Medical Journal, 2, 499.

EVANS, D.S. (1968). Acute dilatation and spontaneous rupture of the stomach. British Journal of Surgery, 55, 940.

JAMIESON, R.A. \& KAY, A.W. (1959). A Textbook of Surgical Physiology, 1, 520. E.S Livingstone: Edinburgh and London.

KEAN, S.B.V., FENNEL, J.S. \& TOMKIN, G.H. (1978). Acute pancreatitis, acute gastric dilatation, duodenal ileus following re-feeding in anorexia nervosa. Irish Journal of Medical Science, 147, 191. pancreatitis with the re-feeding phase in anorexia nervosa (Scobie, 1973; Keane et al. 1978).

Calcification or fibrosis of the pancreas as a cause of diabetes has been observed in many areas of the world $c$. where nutrition is poor. It is usually secondary to $\vec{\Rightarrow}$ chronic relapsing pancreatitis and an early description $\stackrel{\oplus}{\stackrel{9}{+}}$ of this condition noted severe emaciation in each case? (West, 1978). The aetiology of acute pancreatitis itself $\overline{\bar{n}}$. remains uncertain although many clinicians believe a $\frac{\bar{\rho}}{\vec{D}}$ common precipitant to be the reflux of bile into the $\stackrel{\varnothing}{\Omega}$ pancreatic ducts (Jamieson \& Kay, 1959).

A possible sequence of events for this patient could $\vec{\circ}$ be as follows. The introduction of food following a prolonged period of reduced intake and severe emacia- $\vec{\omega}$ tion may lead to gastric dilatation with duodenal ileus. The resultant rise in duodenal pressure may produce 8 reflux into the pancreatic ducts initiating the inflam- 3 . matory reaction. This case provides further evidence 9 of an association between these conditions.

From a clinical point of view, what seems to be $\overrightarrow{\overrightarrow{.}}$ important is that those who are responsible for the care of these patients are aware both of the way in 0 which this complication presents itself and of its $\rightarrow$ timing in relation to the course of the illness. It would $c$ seem to be unwise in this group of patients to assume that these symptoms are either psychological in origin or benign in nature. Continual assessment of the patient's physical state during this critical phase mag $\vec{\emptyset}$ be required to ensure a successful outcome.

\section{Acknowledgement}

I would like to thank Dr J.L. Cox, Consultant Psychiatrist, for permission to report this case.

RUSSELL, G.F.M. (1966). Acute dilatation of the stomach in a patient with anorexia nervosa. British Journal of Psychiatry, 112, 203.

SCOBIE, B.S. (1973). Acute gastric dilatation and duodenal ileus in anorexia nervosa. Medical Journal of Australia, 2, 932.

WEST, K.M. (1978). Epidemiology of Diabetes and its Vascular Lesions. 1, 325. Elsevier North-Holland, New York. 